\title{
Diagnosis and treatment of positional plagiocephaly
}

\author{
Bok Ki Jung, \\ In Sik Yun \\ Department of Plastic and \\ Reconstructive Surgery, Institute for \\ Human Tissue Restoration, Yonsei \\ University College of Medicine, Seoul, \\ Korea
}

\begin{abstract}
Positional plagiocephaly is increasing in infants. Positional plagiocephaly is an asymmetric deformation of skull due to various reasons; first birth, assisted labor, multiple pregnancy, prematurity, congenital muscular torticollis and position of head. Positional plagiocephaly can mostly be diagnosed clinically and by physical examinations. The simplest way to assess the severity of plagiocephaly is to use a diagonal caliper during physical examination, which measures the difference between the diagonal lengths on each side of the head. Plagiocephaly can be treated surgically or conservatively. Positional plagiocephaly, which is not accompanied by craniosynostosis, is treated conservatively. Conservative treatments involve a variety of treatments, such as change of positions, physiotherapy, massage therapy, and helmet therapy. Systematic approaches to clinical examination, diagnosis and treatment of positional plagiocephaly can be necessary and the age-appropriate treatment is recommended for patients with positional plagiocephaly.
\end{abstract}

Keywords: Craniosynostosis / Helmet therapy / Plagiocephaly

\section{INTRODUCTION}

Plagiocephaly can be classified into two types, plagiocephaly that is followed by craniosynostosis and positional plagiocephaly in the absence of craniosynostosis [1]. In case of plagiocephaly not accompanied by craniosynostosis, the sutures of the skull are normal, and the growth of the skull is often not hindered [1-4]. However, the skull becomes asymmetrically distorted due to various reasons. The most common shape of the head is flattened on one side on the posterior part of the head [5-8]. When plagiocephaly is accompanied by craniosynostosis, deformation of the skull is caused by premature fusion of the sutures present in the skull. This condition is often surgically treated [9-13] and helmet therapy may also be performed [14-17].

In the late 1980s, the American Academy of Pediatrics (AAP)

\footnotetext{
Correspondence: In Sik Yun

Department of Plastic and Reconstructive Surgery, Gangnam Severance Hospital, Yonsei University College of Medicine, 211 Eonju-ro, Gangnam-gu, Seoul 06273, Korea

E-mail: eqatom@yuhs.ac
}

Received February 20, 2020 / Revised March 2, 2020 / Accepted March 4, 2020 claimed that sudden infant death syndrome (SIDS) is closely related to the prone sleeping position [3,18]. In April 1992, the AAP recommended and enforced a campaign to encourage every parent to position the infants in the supine position when sleeping to prevent SIDS. Consequently, the incidence of SIDS decreased by more than $40 \%$; however, the incidence of positional plagiocephaly increased by approximately $600 \%$ [18]. The incidence of positional plagiocephaly is increasing every year [19].

Positional plagiocephaly can be defined as deformation and flattening of one side of the head by continuous application of an external force. Such deformation and flattening processes mostly occur in the first few months after birth and are mainly affected by the position of the head $[3,4]$. The increasing number of patients with plagiocephaly and continued interest and demand of the parents have led to new treatments with the range gradually increasing as well. Positional plagiocephaly can be prevented when the parents fully understand the causes of the disease and show continued interest in infant welfare. Studies have reported that educating the parents on the posture and environment of the infant after birth is beneficial for the pre- 
vention of positional plagiocephaly $[3,7,20]$. If positional plagiocephaly is detected at a very early stage (before 2 months after birth) with congenital muscular torticollis, early physical therapy for congenital muscular torticollis is very effective in correcting positional plagiocephaly. Furthermore, maintaining a position that does not exert pressure on the conservatively flat part of the head or a placing the infant in the prone position may be helpful $[21,22]$. Following the report of helmet therapy by Clarren et al. [23] in 1972, several studies have advocated that helmet therapy is very effective as a supplementary treatment for infants with severe positional plagiocephaly.

\section{CAUSES}

Risk factors for positional plagiocephaly include first birth, assisted labor, multiple pregnancy, injuries from birth, congenital malformation, premature infants, boys, and especially congenital muscular torticollis and remaining in the prone position for a prolonged time $[4,7,23,24]$.

The most common cause of the disease is the deformation of the head shape during birth that is not corrected due to infant being placed in the same sleeping position. During birth, the skull rapidly changes in shape due to the effect of gravity, fluidity of the cranial sutures, and plasticity of the brain. This allows the relatively large head to exit the narrow birth canal. In most infants, the deformed skull naturally corrects itself over time [1$4,7]$. However, most infants sleep in the supine position and turn their heads in response to certain stimuli. In conditions, such as congenital muscular torticollis, the tense neck muscle causes the infants to hold their neck and head in a preferred position [21,22]. Maintaining the head in the same position for a prolonged period will cause flattening of the part of the head that is facing the floor, and the upper part of the face will begin to flatten thereafter. Both sides of the head will gradually deform, leading to an asymmetrical shape of the head [7]. When a part of the skull becomes flat, the infant's head naturally turns towards the flat side and is pulled by gravity. The degree of flattening gradually worsens during the first 5-6 months after birth. Compensatory asymmetry of the skull is observed as the skull assumes the shape of a parallelogram. Consequently, the positions of the ear, lower jaw, and eye pit change, leading to facial asymmetry $[7,25]$. If one side of the back of the head is severely flattened, the occipital bone is pushed towards the opposite side on the back of the head, causing the opposite occipital bone to protrude prominently. Greater force of gravity is applied to regions with a larger surface area. As the frontal bone on the opposite side of the occipital bone flattened by gravity becomes flat, the ipsilateral frontal bone protrudes in a manner similar to the occipital bone. Therefore, the head tilts towards the flattened occipital bone and the position of the ears changes

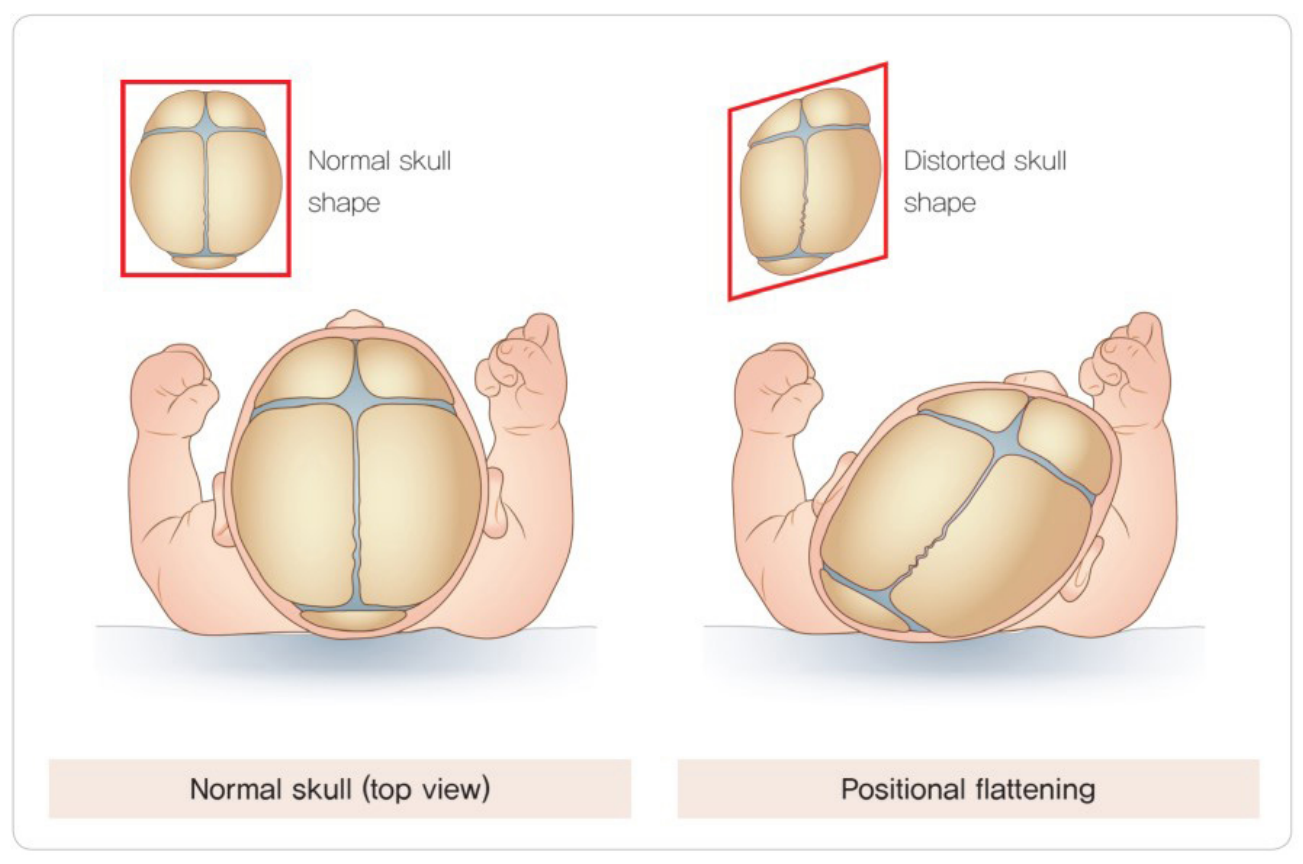

Fig. 1. Mechanism underlying the development of positional plagiocephaly. When a portion of the skull becomes flat, the infant's head is naturally turned towards the flat part and is pulled back by gravity. The degree of flattening gradually increases, and compensatory asymmetry of the skull is observed. Eventually, the skull is shaped like a parallelogram, and the positions of the ear, lower jaw, and eye pit change, thus, leading to facial asymmetry. Reprinted with permission from the Korean Pediatric Neurosurgical Society (the Korean Neurosurgical Society) [26]. 
(Fig. 1) $[3,7,25,26]$. In cases of multiple pregnancy, the limited intrauterine space prevents fetal posture change. This can lead to the application of constant force on the fetus's head, leading to positional plagiocephaly $[3,7,19]$. Positional plagiocephaly is often accompanied by congenital muscular torticollis, which may contribute towards the development positional plagiocephaly due to the neck position and tension in the neck muscles and ligaments [21,22].

Such progression of asymmetry terminates once the infant begins to move, change positions during sleeping, and stand. The deformity of the skull can be naturally corrected over a period of a few months. However, the degree of natural correction varies depending on the severity of asymmetry, and full correction is rarely observed. The uncorrected asymmetrical shape of the head shape at this point is mostly maintained throughout adulthood [3,7,25].

\section{DIAGNOSIS}

Plagiocephaly can mostly be diagnosed clinically and by physical examinations. Radiography of the skull is performed to determine craniosynostosis, and ultrasound, magnetic resonance imaging, and computed tomography can be performed to achieve a definitive diagnosis. Moreover, three-dimensional (3D) reconstitution of the skull after imaging may also aid in detecting craniosynostosis [7,25].

The simplest way to assess the severity of plagiocephaly is to use a diagonal caliper during physical examination, which measures the difference between the diagonal lengths on each side of the head. A diagonal caliper is a cost-effective tool; however, there may be errors between the measurements, which can reduce the accuracy of evaluating the severity of plagiocephaly. Moreover, the difference between the diagonal lengths may be reduced by the scalp or hair of the patient, and the error can be large when the patient markedly when the measurements are being recorded. A study reported that errors in the readings recorded by different examiners may be as high as $2.2 \mathrm{~mm}$ on an average and that imaging data can aid in reducing such errors when measuring the difference in the diagonal length $[27,28]$.

Difference of 9-12 $\mathrm{mm}$ and more than $12 \mathrm{~mm}$ in the cranial vault asymmetry are defined as mild to moderate and severe asymmetry, respectively $[27,28]$. The cranial vault asymmetry index is used to determine the severity of positional plagiocephaly and can be calculated as shown below. An index $>3.5$ indicates severe asymmetry [27-29].

Cranial vault asymmetry index $=$

Difference between cranial diagonal diameter

Shorter cranial diagonal diameter

\section{TREATMENT}

Plagiocephaly can be treated surgically or conservatively. Plagiocephaly, accompanied by craniosynostosis, is often surgically treated [9-13]. Positional plagiocephaly, which is not accompanied by craniosynostosis, is treated conservatively [14-17].

Conservative treatments involve a variety of treatments, such as change of positions [7], physiotherapy [30], massage therapy [7], and helmet therapy [30-33]. Promising outcomes can be achieved from conservative treatments when the treatments are initiated at an early stage of the disease due to the plasticity of the cranium and cranial sutures of the infants [7,30-33]. Furthermore, confirmation of other causes of positional plagiocephaly and treatment of possible congenital deformity are required. Performing physiotherapy for the tense neck muscles may be beneficial for congenital muscular torticollis [30].

\section{Principles of the helmet treatment}

Many studies have reported the use of external braces for the treatment of positional plagiocephaly. External braces include helmet orthosis, cranial orthosis, cranial orthotic device, and orthotic headbands, which are known to be very effective for the correction of the asymmetrical shape of the head [30-34]. The helmet generally covers the entire head like a bicycle helmet. The headband covers the entire head, except for the top of the head. Regardless of the design, customized head braces for patients are made from high-temperature thermoplastic material (Surlyn, Dupont, Wilmington, DE, USA) using high density hypoallergenic medical grade foam (Fig. 2) [26,34-36]. First, the head of the patient is scanned with a $3 \mathrm{D}$ laser to create a head model.

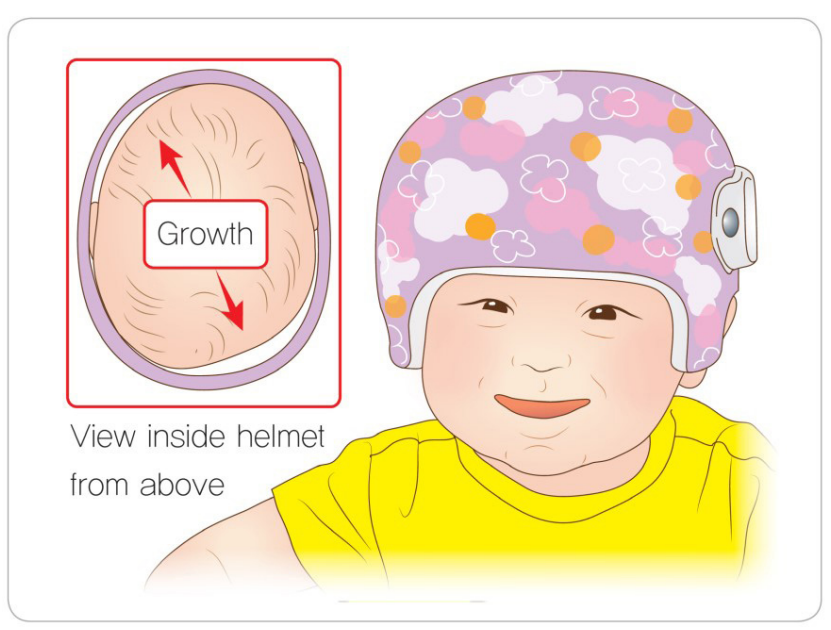

Fig. 2. Orthotic helmet. The helmet is shaped to cover the entire head, while the headband covers all parts of the head, except the top of the head. High density hypoallergenic medical grade foam is used to make the helmet. Reprinted with permission from the Korean Pediatric Neurosurgical Society (the Korean Neurosurgical Society) [26]. 
The helmet is so that the protruding portion of the head fits tight and there is empty space around the flat part to aid the head in assuming a symmetrical and typical shape. As the patient's skull grows, the growth of the protruding part of the head is limited, while the extra space around the flat portion of the head allows for more growth towards the relatively less flat part of the head with less resistance [37]. Some studies suggest that faster correction can be achieved by applying pressure on the protruding portion of the head, which is actively growing [34-39].

\section{Application of helmet treatment}

There is controversy regarding the age at which helmet treatment should be initiated. If positional plagiocephaly is discovered at an early stage, change of position is often the main method of treatment with further observation. If mild positional plagiocephaly is observed before 4 months of age, changing the sleeping position of the infant should be started early [34]. The minimum age of patients eligible for helmet treatment is not defined, and helmet treatment should be initiated before the infants can control their head movements $[38,40]$. Helmet treatment is more effective if it is performed at an early stage of development of the head, and the treatment should begin when the skull is rapidly growing. Therefore, most doctors recommend initiating helmet treatment before 6 months of age for rapid and effective correction of the head. When positional plagiocephaly is not corrected or improved on conservative treatment, helmet treatment should be initiated for babies at the age of 6 months [34,41-43]. Approximately 85\% of cranial growth is achieved in the first 12 months after birth, and the cranial growth rate is significantly decreased from 12 months until 24 months of age. Therefore, helmet treatment is very effective up until 12 months of age, and the effect is very weak if the treatment is started after 12 months of age. The outcome of asymmetry correction by skull growth is very poor after 18 months of age [34,41-44].

There are three reasons for the controversy regarding the age at which helmet treatment should be initiated. First, the degree of asymmetry of the head determines the feasibility of helmet treatment. Therefore, variation in the results according to the degree of asymmetry will be apparent, regardless of the timing of the helmet treatment. Second, most patients who receive helmet treatment also receive conservative treatment, such as changing the posture, which limits the accurate determination of the effect of helmet treatment. Third, the duration of treatment and pressure exerted by the helmet vary according to the physicians and the type of helmet used. Thus, defining the appropriate age to start helmet therapy is challenging.

However, most studies report that better results are obtained
Table 1. Severity of positional plagiocephaly and recommendations for the time of initiating helmet treatment

\begin{tabular}{ll}
\hline Age & Treatments for positional plagiocephaly \\
\hline Before 4 months & $\begin{array}{l}\text { Posture change }[34,41,42] \\
\text { Physical therapy for congenital muscular torticollis [34] }\end{array}$ \\
4-6 Months & $\begin{array}{c}\text { For mild to moderate positional plagiocephaly, continue } \\
\text { posture change [34] } \\
\text { For severe positional plagiocephaly, initiate helmet therapy } \\
{[34,43]}\end{array}$ \\
Older than 6 months & $\begin{array}{c}\text { For mild to moderate positional plagiocephaly with no } \\
\text { improvements after posture change, initiate helmet } \\
\text { therapy [34,42] } \\
\text { For severe positional plagiocephaly, initiate helmet therapy } \\
{[34,42]}\end{array}$ \\
\hline
\end{tabular}

when helmet therapy begins sooner. In particular, many studies also report that the effect of helmet therapy is stronger when helmet therapy is performed in combination with posture changing and physiotherapy for congenital torticollis. Therefore, age-appropriate treatment is recommended for patients with positional plagiocephaly (Table 1) [34,41-43]. Once helmet therapy is initiated, it is fundamental that the parents are informed that the patients must not wear the helmet for more than 20 hours a day. The total duration of helmet treatment averages between 2 to 6 months, and the parents should note that the duration will vary depending on the degree of asymmetry of the head shape and the time of commencement of treatment. Promising results can be obtained when the parents of the patient are interested in the head shape of the patient and actively participate in implementation of helmet therapy. The parents should be informed that correction of the shape of the head with active helmet treatment can also prevent facial asymmetry, with the growth of the skull [7,30,39,45-47].

\section{Complications and limitations of helmet therapy}

The complications of helmet therapy, although very rare, include (1) inadequate correction due to a poorly fitting helmet, (2) damage to the skin at the site of application of pressure by the helmet, (3) damage to the scalp and temporary hair loss at the site of application of pressure by the helmet, (4) contact allergic reaction of the skin depending on the material of the helmet. Most complications, including skin and scalp damage, temporary hair loss, and allergic reactions, are naturally cured without any special treatment and can be prevented if the parents pay attention. The complications arising from poorly fitting helmets can also be prevented by modifying the helmet through parental attention and regular outpatient care [31,48-50]. However, helmet treatment has economic disadvantages compared to other conservative treatments $[34,40,48,51]$. The cost of the helmet varies with the type of the helmet; however, the helmet 
costs approximately $\$ 2,000-\$ 3,000$ and 3 million won in the United States and Korea, respectively. Only a small part of the cost is covered by insurance, and helmet treatment is not economically effective compared to posture change. Furthermore, follow-up of the patients' head shape and modification of the helmet between treatments are necessary, which will incur additional costs. In addition, there is a lack of studies on the longterm follow-up of self-satisfaction and cosmetic aspects of asymmetry remaining after adolescence and adulthood.

Plagiocephaly accompanied by craniosynostosis and helmet treatment (helmet treatment after surgery)

Surgical treatment is very effective in cases of plagiocephaly with craniosynostosis, and various surgical methods are available [12,52-55]. However, many studies report that, since there are many cases of recurrence and changes in the shape of the skull after surgery, helmet treatment after surgical treatment, such as craniotomy and distraction osteogenesis of the cranium, is beneficial $[7,14,17,55-58]$. Helmet treatment after surgery does not limit cranial growth and shows excellent functional and cosmetic results in remodeling of the skull shape. Postoperative helmet treatment can aid in allowing the skull and brain grow normally. The average duration of the treatment is 6-12 months and the treatment is generally continued till the age of $12-18$ months $[7,14,17,55-58]$.

\section{CONCLUSION}

Positional plagiocephaly is increasing in infants for various reasons. It can mostly be diagnosed clinically and by physical examinations, and treated surgically or conservatively. Most of positional plagiocephaly, which is not accompanied by craniosynostosis, is treated conservatively. The helmet therapy has controversy as to when to start and how long to maintain the therapy. However, for the patients with positional plagiocephaly, appropriate helmet therapy, in accordance with their ages, is a very effective treatment to change the shape and position of their head.

\section{NOTES}

\section{Conflict of interest}

No potential conflict of interest relevant to this article was reported.

\section{ORCID}

Bok Ki Jung https://orcid.org/0000-0002-4347-560X

In Sik Yun

\section{REFERENCES}

1. Bennis Y, Wolber A, Vinchon M, Belkhou A, DuquennoyMartinot V, Guerreschi P. Non syndromic craniosynostosis. Ann Chir Plast Esthet 2016;61:389-407.

2. Feijen MM, Claessens EA, Dovens AJ, Vles JS, van der Hulst RR. Babies with cranial deformity. Ned Tijdschr Geneeskd 2009;153:A368.

3. Argenta LC, David LR, Wilson JA, Bell WO. An increase in infant cranial deformity with supine sleeping position. J Craniofac Surg 1996;7:5-11.

4. Najarian SP. Infant cranial molding deformation and sleep position: implications for primary care. J Pediatr Health Care 1999; 13:173-7.

5. Christensen L, Ostergaard JR, Norholt SE. Positional plagiocephaly. Ugeskr Laeger 2002;165:46-50.

6. Hummel P, Fortado D. Impacting infant head shapes. Adv Neonatal Care 2005;5:329-40.

7. Morrison CS, Chariker M. Positional plagiocephaly: pathogenesis, diagnosis, and management. J Ky Med Assoc 2006;104: 136-40.

8. Pivar SJ, Scheuerle A. Variable co-diagnosis of plagiocephaly and torticollis in Texas health care facilities. J Craniofac Surg 2006;17:236-40.

9. Sgouros S, Goldin JH, Hockley AD, Wake MJ. Posterior skull surgery in craniosynostosis. Childs Nerv Syst 1996;12:727-33.

10. Holmes AD, Wright GW, Meara JG, Heggie AA, Probert TC. LeFort III internal distraction in syndromic craniosynostosis. J Craniofac Surg 2002;13:262-72.

11. Meier PM, Goobie SM, DiNardo JA, Proctor MR, Zurakowski D, Soriano SG. Endoscopic strip craniectomy in early infancy: the initial five years of anesthesia experience. Anesth Analg 2011;112:407-14.

12. Hassanpour SE, Abbasnezhad M, Alizadeh Otaghvar H, Tizmaghz A. Surgical correction of unicoronal craniosynostosis with frontal bone symmetrization and staggered osteotomies. Plast Surg Int 2018;2018:3793592.

13. Esparza J, Hinojosa J, Garcia-Recuero I, Romance A, Pascual B, Martinez de Aragon A. Surgical treatment of isolated and syndromic craniosynostosis. Results and complications in 283 consecutive cases. Neurocirugia (Astur) 2008;19:509-29.

14. Law J, Marucci DD, Gates RJ, Fowler A. Unilateral pterional polycraniosynostosis treated with craniectomy and helmet therapy. Plast Reconstr Surg Glob Open 2017;5:e1245.

15. de Jong G, Tolhuisen M, Meulstee J, van der Heijden F, van Lindert E, Borstlap W, et al. Radiation-free 3D head shape and volume evaluation after endoscopically assisted strip craniectomy followed by helmet therapy for trigonocephaly. J Cranio- 
maxillofac Surg 2017;45:661-71.

16. Ridgway EB, Berry-Candelario J, Grondin RT, Rogers GF, Proctor MR. The management of sagittal synostosis using endoscopic suturectomy and postoperative helmet therapy. J Neurosurg Pediatr 2011;7:620-6.

17. Chong S, Wang KC, Phi JH, Lee JY, Kim SK. Minimally invasive suturectomy and postoperative helmet therapy: advantages and limitations. J Korean Neurosurg Soc 2016;59:227-32.

18. Pelligra R, Doman G, Leisman G. A reassessment of the SIDS back to sleep campaign. ScientificWorldJournal 2005;5:550-7.

19. Gonzalez de Dios J, Moya M, Jimenez L, Alcala-Santaella R, Carratala F. Increase in the incidence of occipital plagiocephaly. Rev Neurol 1998;27:782-4.

20. Neufeld S, Birkett S. Positional plagiocephaly: a community approach to prevention and treatment. Alta RN 1999;55:15-6.

21. Graham T, Gilbert N, Witthoff K, Gregory T, Walsh M. Significant factors influencing the effectiveness of cranial remolding orthoses in infants with deformational plagiocephaly. J Craniofac Surg 2019;30:1710-3.

22. Stellwagen L, Hubbard E, Chambers C, Jones KL. Torticollis, facial asymmetry and plagiocephaly in normal newborns. Arch Dis Child 2008;93:827-31.

23. Clarren SK, Smith DW, Hanson JW. Helmet treatment for plagiocephaly and congenital muscular torticollis. J Pediatr 1979; 94:43-6.

24. Ditthakasem K, Kolar JC. Deformational plagiocephaly: a review. Pediatr Nurs 2017;43:59-64.

25. Rogers GF. Deformational plagiocephaly, brachycephaly, and scaphocephaly. Part I: terminology, diagnosis, and etiopathogenesis. J Craniofac Surg 2011;22:9-16.

26. The Korean Pediatric Neurosurgical Society (the Korean Neurosurgical Society). Textbook of pediatric neurosurgery. Seoul: Medbook; 2018.

27. Zonenshayn M, Kronberg E, Souweidane MM. Cranial index of symmetry: an objective semiautomated measure of plagiocephaly. Technical note. J Neurosurg 2004;100(5 Suppl Pediatrics):537-40.

28. Aarnivala HE, Valkama AM, Pirttiniemi PM. Cranial shape, size and cervical motion in normal newborns. Early Hum Dev 2014;90:425-30.

29. Argenta L, David L, Thompson J. Clinical classification of positional plagiocephaly. J Craniofac Surg 2004;15:368-72.

30. Di Chiara A, La Rosa E, Ramieri V, Vellone V, Cascone P. Treatment of deformational plagiocephaly with physiotherapy. J Craniofac Surg 2019;30:2008-13.

31. Clarren SK. Plagiocephaly and torticollis: etiology, natural history, and helmet treatment. J Pediatr 1981;98:92-5.

32. Vles JS, Colla C, Weber JW, Beuls E, Wilmink J, Kingma H.
Helmet versus nonhelmet treatment in nonsynostotic positional posterior plagiocephaly. J Craniofac Surg 2000;11:572-4.

33. van Wijk RM, van Vlimmeren LA, Groothuis-Oudshoorn CG, Van der Ploeg CP, Ijzerman MJ, Boere-Boonekamp MM. Helmet therapy in infants with positional skull deformation: randomised controlled trial. Version 2. BMJ 2014;348:g2741.

34. Mortenson P, Steinbok P, Smith D. Deformational plagiocephaly and orthotic treatment: indications and limitations. Childs Nerv Syst 2012;28:1407-12.

35. Ripley CE, Pomatto J, Beals SP, Joganic EF, Manwaring KH, Moss SD. Treatment of positional plagiocephaly with dynamic orthotic cranioplasty. J Craniofac Surg 1994;5:150-9.

36. de Ribaupierre S, Vernet O, Rilliet B, Cavin B, Kalina D, Leyvraz PF. Posterior positional plagiocephaly treated with cranial remodeling orthosis. Swiss Med Wkly 2007;137:368-72.

37. Loveday BP, de Chalain TB. Active counterpositioning or orthotic device to treat positional plagiocephaly? J Craniofac Surg 2001;12:308-13.

38. Robinson S, Proctor M. Diagnosis and management of deformational plagiocephaly. J Neurosurg Pediatr 2009;3:284-95.

39. Rogers GF. Deformational plagiocephaly, brachycephaly, and scaphocephaly. Part II: prevention and treatment. J Craniofac Surg 2011;22:17-23.

40. Lee A, Van Pelt AE, Kane AA, Pilgram TK, Govier DP, Woo AS, et al. Comparison of perceptions and treatment practices between neurosurgeons and plastic surgeons for infants with deformational plagiocephaly. J Neurosurg Pediatr 2010;5:36874.

41. Kennedy E, Majnemer A, Farmer JP, Barr RG, Platt RW. Motor development of infants with positional plagiocephaly. Phys Occup Ther Pediatr 2009;29:222-35.

42. Graham JM Jr, Kreutzman J, Earl D, Halberg A, Samayoa C, Guo X. Deformational brachycephaly in supine-sleeping infants. J Pediatr 2005;146:253-7.

43. McGarry A, Dixon MT, Greig RJ, Hamilton DR, Sexton S, Smart H. Head shape measurement standards and cranial orthoses in the treatment of infants with deformational plagiocephaly. Dev Med Child Neurol 2008;50:568-76.

44. Xia JJ, Kennedy KA, Teichgraeber JF, Wu KQ, Baumgartner JB, Gateno J. Nonsurgical treatment of deformational plagiocephaly: a systematic review. Arch Pediatr Adolesc Med 2008;162: 719-27.

45. Katzel EB, Koltz PF, Sbitany H, Girotto JA. Treatment of plagiocephaly with helmet molding therapy: do actual results mimic perception? Cleft Palate Craniofac J 2011;48:205-9.

46. Kunz F, Schweitzer T, Grobe S, Wabmuth N, Stellzig-Eisenhauer A, Bohm $\mathrm{H}$, et al. Head orthosis therapy in positional plagiocephaly: longitudinal 3D-investigation of long-term out- 
comes, compared with untreated infants and with a control group. Eur J Orthod 2019;41:29-37.

47. Steinberg JP, Rawlani R, Humphries LS, Rawlani V, Vicari FA. Effectiveness of conservative therapy and helmet therapy for positional cranial deformation. Plast Reconstr Surg 2015;135: 833-42.

48. Gump WC, Mutchnick IS, Moriarty TM. Complications associated with molding helmet therapy for positional plagiocephaly: a review. Neurosurg Focus 2013;35:E3.

49. Wilbrand JF, Wilbrand M, Malik CY, Howaldt HP, Streckbein P, Schaaf $\mathrm{H}$, et al. Complications in helmet therapy. J Craniomaxillofac Surg 2012;40:341-6.

50. Teichgraeber JF, Ault JK, Baumgartner J, Waller A, Messersmith M, Gateno J, et al. Deformational posterior plagiocephaly: diagnosis and treatment. Cleft Palate Craniofac J 2002;39: 582-6.

51. Sheu SU, Ethen MK, Scheuerle AE, Langlois PH. Investigation into an increase in plagiocephaly in Texas from 1999 to 2007. Arch Pediatr Adolesc Med 2011;165:708-13.

52. Elbanoby T. Craniofacial distraction in the management of anterior plagiocephaly: a novel idea and a systematic review of the literature. Pediatr Neurosurg 2018;53:193-9.
53. Sufianov AA, Gaibov SS, Sufianov RA. Surgical aspects of endoscopic treatment of sagittal craniosynostosis (scaphocephaly) in children. Zh Vopr Neirokhir Im N N Burdenko 2013;77: 26-34.

54. Bonfield CM, Lee PS, Adamo MA, Pollack IF. Surgical treatment of sagittal synostosis by extended strip craniectomy: cranial index, nasofrontal angle, reoperation rate, and a review of the literature. J Craniomaxillofac Surg 2014;42:1095-101.

55. Delye HHK, Borstlap WA, van Lindert EJ. Endoscopy-assisted craniosynostosis surgery followed by helmet therapy. Surg Neurol Int 2018;9:59.

56. Chou PY, Hallac RR, Patel S, Cho MJ, Stewart N, Smartt JM, et al. Three-dimensional changes in head shape after extended sagittal strip craniectomy with wedge ostectomies and helmet therapy. J Neurosurg Pediatr 2017;19:684-9.

57. Rottgers SA, Lohani S, Proctor MR. Outcomes of endoscopic suturectomy with postoperative helmet therapy in bilateral coronal craniosynostosis. J Neurosurg Pediatr 2016;18:281-6.

58. Jimenez DF, Barone CM, McGee ME. Design and care of helmets in postoperative craniosynostosis patients: our personal approach. Clin Plast Surg 2004;31:481-7. 